\title{
The tumor-suppressive microRNA-143/145 cluster inhibits cell migration and invasion by targeting GOLM1 in prostate cancer
}

\author{
Satoko Kojima ${ }^{1,4}$, Hideki Enokida ${ }^{2,4}$, Hirofumi Yoshino ${ }^{2}$, Toshihiko Itesako ${ }^{2}$, Takeshi Chiyomaru ${ }^{2}$, \\ Takashi Kinoshita $^{3}$, Miki Fuse ${ }^{3}$, Rika Nishikawa ${ }^{3}$, Yusuke Goto ${ }^{3}$, Yukio Naya ${ }^{1}$, Masayuki Nakagawa ${ }^{2}$ \\ and Naohiko Seki ${ }^{3}$
}

Our recent study of microRNA (miRNA) expression signature of prostate cancer (PCa) has revealed that the microRNA-143/145 ( $m i R-143 / 145$ ) cluster is significantly downregulated in cancer tissues, suggesting that these cluster miRNAs are candidate tumor suppressors. The aim of this study was to investigate the functional significance of the miR-143/145 cluster in PCa cells and to identify novel targets regulated by these cluster miRNAs in PCa. Restoration of miR-143 or miR-145 in PCa cell lines (PC3 and DU145) revealed that these miRNAs significantly inhibited cancer cell migration and invasion. Gene expression data and in silico analysis demonstrated that Golgi membrane protein 1 (GOLM1) resembling a type II golgi transmembrane protein was a potential target of $m i R-143 / 145$ cluster target gene. Gene expression studies and luciferase reporter assays showed that GOLM1 was directly regulated by the miR-143/145 cluster. Silencing of GOLM1 resulted in significant inhibition of cell migration and invasion in PCa cells. Furthermore, the expression of GOLM1 was upregulated in cancer tissues by immunohistochemistry. Loss of the tumor-suppressive miR-143/145 cluster enhanced cancer cell migration and invasion in PCa through directly regulating GOLM1. Our data on target genes regulated by the tumor-suppressive miR-143/145 cluster provide new insights into the potential mechanisms of PCa oncogenesis and metastasis. Journal of Human Genetics (2014) 59, 78-87; doi:10.1038/jhg.2013.121; published online 28 November 2013

Keywords: GOLM1; microRNA; miR-143; miR-145; prostate cancer; tumor suppressor

\section{INTRODUCTION}

Prostate cancer (PCa) is the most frequently diagnosed cancer and the second leading cause of cancer death among men in developed countries. ${ }^{1}$ Most patients are initially responsive to androgendeprivation therapy, but their cancers eventually become resistant to androgen-deprivation therapy and progress to castration-resistant prostate cancer (CRPC). Currently, CRPC is difficult to treat, and most clinical trials for advanced PCa have shown limited benefits, with disease progression and metastasis to the bone or other sites. ${ }^{2,3}$ Therefore, understanding the molecular mechanisms of CRPC and metastatic pathways underlying $\mathrm{PCa}$ using currently available genomic approaches would help to improve therapies for and prevention of the disease.

The discovery of non-coding RNAs (ncRNAs) in the human genome was an important conceptual breakthrough in the postgenome sequencing era. ${ }^{4}$ Improving our understanding of ncRNAs is necessary for continued progress in cancer research. MicroRNAs (miRNAs) are endogenous small ncRNA molecules (19-22 bases in length) that regulate protein-coding gene expression by repressing translation or cleaving RNA transcripts in a sequence-specific manner. Currently, 2042 human mature miRNAs are registered at miRBase release 19.0 (http://microrna.sanger.ac.uk/). miRNAs are unique in their ability to regulate multiple protein-coding genes. Bioinformatic predictions indicate that miRNAs regulate $>30-60 \%$ of the proteincoding genes in the human genome. ${ }^{5,6}$

Emerging evidence has demonstrated that miRNAs are aberrantly expressed in many human cancers and have significant roles in the initiation, development and metastasis of these cancers. ${ }^{7,8}$ It is believed that normal regulatory mechanisms can be disrupted by the aberrant expression of tumor suppressive or oncogenic miRNAs in cancer cells. Therefore, identification of aberrantly expressed miRNAs is the first step toward elucidating miRNA-mediated oncogenic pathways. Based on this, we identified miRNA expression signature in PCa using clinical specimens and found that miR-145 expression was significantly downregulated in $\mathrm{PCa} .{ }^{9}$ We also reported that miR-145 suppresses cell proliferation, migration and invasion by

${ }^{1}$ Department of Urology, Teikyo University Chiba Medical Center, Chiba, Japan; ${ }^{2}$ Department of Urology, Graduate School of Medical and Dental Sciences, Kagoshima University, Kagoshima, Japan and ${ }^{3}$ Department of Functional Genomics, Graduate School of Medicine, Chiba University, Chiba, Japan

${ }^{4}$ These authors contributed equally to this work.

Correspondence: Dr N Seki, Department of Functional Genomics, Graduate School of Medicine, Chiba University, 1-8-1 Inohana Chuo-ku, Chiba 260 8670, Japan. E-mail: naoseki@faculty.chiba-u.jp

Received 21 September 2013; revised 28 October 2013; accepted 28 October 2013; published online 28 November 2013 
targeting FSCN1. ${ }^{10,11}$ Other studies have reported that the miR-143/ 145 cluster is significantly downregulated in PCa and have suggested its important association with oncogenesis and bone metastasis in PCa. ${ }^{12,13}$

The aim of the present study was to investigate the functional significance of the miR-143/145 cluster and to identify the molecular targets regulated by this cluster in PCa cells. Genome-wide gene expression data and in silico database analysis showed that Golgi membrane protein 1 (GOLM1) was a promising candidate target of the miR-143/145 cluster. GOLM1, also known as Golgi phosphoprotein 2 (GOLPH2) and Golgi protein 73 (GP73), is a newly identified Golgi-associated protein. Although the functional roles of GOLM1 are currently unclear, GOLM1 has been shown to interact with other proteins through its transmembrane protein domain. ${ }^{14}$ The overexpression of GOLM1 has been reported in several types of cancers, including $\mathrm{PCa} .{ }^{15-17}$ Elucidation of the molecular targets regulated by the tumor-suppressive miR-143/145 cluster will provide new insights into the potential molecular mechanisms of PCa oncogenesis and metastasis and will facilitate the development of novel therapeutic strategies for the treatment of PCa.

\section{MATERIALS AND METHODS}

\section{Clinical prostate specimens}

Clinical specimens were obtained from patients at the Teikyo University Chiba Medical Center from 2008 to 2013. All the patients had elevated levels of prostate-specific antigen and had undergone transrectal prostate needle biopsy. Non-cancerous prostate tissue $(n=24)$ was obtained from patients who were negative for malignancy without indurations on the prostate. Prostate tumor tissues $(n=28)$ contained $90-100 \%$ malignant cells in the biopsy cores. Patients' characteristics are shown in Table 1. Before tissue collection, all patients provided written informed consent of tissue donation for research purposes. The protocol was approved by the Institutional Review Board of Teikyo University.

\section{Cell culture}

PC3 and DU145 cells, human PCa cells obtained from the American Type Culture Collection (Manassas, VA, USA), were maintained in RPMI-1640 medium supplemented with $10 \%$ fetal bovine serum in a humidified atmosphere of $5 \% \mathrm{CO}_{2}$ and $95 \%$ air at $37^{\circ} \mathrm{C}$.

\section{RNA extraction}

Total RNA was isolated using TRIzol reagent (Invitrogen, Carlsbad, CA, USA) according to the manufacturer's protocol. RNA concentrations were determined spectrophotometrically, and molecular integrity was checked by gel electrophoresis. RNA quality was confirmed using an Agilent 2100 Bioanalyzer (Agilent Technologies, Santa Clara, CA, USA).

\section{Quantitative real-time reverse transcriptase-PCR (RT-PCR)}

First-strand cDNA was synthesized from $1 \mu \mathrm{g}$ total RNA using a High Capacity cDNA Reverse Transcription Kit (Applied Biosystems, Foster City, CA, USA). Gene-specific PCR products were assayed continuously using a 7900-HT RealTime PCR System (Applied Biosystems) according to the manufacturer's protocol. The initial PCR step consisted of a 10 -min hold at $95^{\circ} \mathrm{C}$, followed by 40 cycles consisting of a $15-\mathrm{s}$ denaturation at $95^{\circ} \mathrm{C}$ and a 1-min annealing/ extension at $63{ }^{\circ} \mathrm{C}$. TaqMan probes and primers for GOLM1 (P/N: Hs00213061_ml) and GAPDH (P/N: Hs02758991_g1) as an internal control were obtained from Applied Biosystems (Assay-On-Demand Gene Expression Products). The expression levels of miR-143 (Assay ID: 002249) and miR-145 (Assay ID: 002278) were analyzed by TaqMan quantitative real-time PCR (TaqMan MicroRNA Assay; Applied Biosystems) and normalized to the expression of RNU48 (Assay ID: 001006). The delta delta Ct method was used to calculate the relative quantities of target genes. All reactions were performed in triplicate, and each assay included negative control reactions that lacked cDNA.
Transfections with mature miRNA and small interfering RNA (siRNA) The following mature miRNA species were used in this study: Pre-miR miRNA Precursor for hsa-miR-143 (Product ID: PM10883) and hsa-miR-145 (Product ID: PM11480; Applied Biosystems). The following siRNAs were used: Stealth Select RNAi siRNA, si-GOLM1 (Cat no. HSS181966 and HSS181968; Invitrogen), and negative control miRNA/siRNA (P/N: AM17111; Applied Biosystems). RNAs were incubated with OPTI-MEM (Invitrogen) and Lipofectamine RNAiMax reagent (Invitrogen) as described previously. The transfection efficiency of miRNA in PC3 and DU145 cells was confirmed based on downregulation of TWF1 (PTK9) mRNA following transfection with miR-1 as previously reported.

\section{Cell proliferation, migration and invasion assays}

Cells were transfected with $10 \mathrm{~nm}$ miRNA or siRNA by reverse transfection and plated in 96-well plates at $3 \times 10^{3}$ cells per well. After $72 \mathrm{~h}$, cell proliferation was determined with the XTT assay using a Cell Proliferation Kit II (Roche Molecular Biochemicals, Mannheim, Germany) as previously reported. ${ }^{18,19}$

Cell migration was evaluated with a wound-healing assay. Cells were plated in six-well plates, and the cell monolayers were scraped using a P-20 micropipette tip. The initial gap length $(0 \mathrm{~h})$ and the residual gap length at $24 \mathrm{~h}$ after wounding were calculated from photomicrographs.

A cell invasion assay was carried out using modified Boyden chambers containing Transwell-precoated Matrigel membrane filter inserts with $8-\mu \mathrm{m}$ pores in 24-well tissue culture plates (BD Biosciences, Bedford, MA, USA) at $2 \times 10^{5}$ cells per well. Cells were transfected with $10 \mathrm{~nm}$ miRNA or siRNA by reverse transfection and plated in $10-\mathrm{cm}$ dishes at $8 \times 10^{5}$ cells per dish. After $48 \mathrm{~h}$, the cells were collected, and $2 \times 10^{5}$ cells were added to the upper chamber of each migration well. Cells were allowed to invade for $48 \mathrm{~h}$. After gentle removal of the non-migratory cells from the filter surface of the upper chamber, the cells that invaded into the lower chamber were fixed and stained with Diff-Quick (Sysmex Corporation, Kobe, Japan). The number of cells that migrated to the lower surface was determined microscopically by counting four areas of constant size per well.

All experiments were performed in triplicate.

\section{Western blotting}

Cells were harvested $72 \mathrm{~h}$ after transfection, and lysates were prepared. Fifty micrograms of protein lysate was separated on Mini-PROTEAN TGX gels (Bio-Rad, Hercules, CA, USA) and transferred to polyvinylidene difluoride membranes. Immunoblotting was performed with rabbit anti-GOLM1 antibodies (1:500; HPA010638, Sigma-Aldrich, St Louis, MO, USA) and antiGAPDH antibodies (1:1000; ab8245, Abcam, Cambridge, UK), used as an internal loading control. Membranes were washed and incubated with antimouse immunoglobulin G horseradish peroxidase-linked antibodies (No. 7076, Cell Signaling Technology, Danvers, MA, USA). Complexes were visualized with an Immun-Star WesternC Chemiluminescence Kit (Bio-Rad), and the expression levels of these genes were evaluated by the ImageJ software provided by National Institutes of Health (NIH), Bethesda, MD, USA (ver.1.44; http://rsbweb.nih.gov/ij/).

Genome-wide gene expression and in silico analyses for the identification of genes regulated by the miR-143/145 cluster

To gain further insight into which genes were affected by the miR-143/145 cluster, we performed a combination of in silico and genome-wide gene expression analyses. First, genes regulated by the miR-143/145 cluster were listed using the TargetScan database as described previously..$^{18,19}$ To investigate the expression status of candidate of miR-143/145 cluster-target genes in PCa clinical specimens, we examined the gene expression profiles in the GEO database (accession number: GSE29079). In this expression study, tissue samples from $47 \mathrm{PCa}$ with clinical high-risk tumors were included, and none of the patients had been treated with neo-adjuvant treatments, such as radiation, cytotoxic and endocrine therapies. ${ }^{20}$

To identify molecular pathways regulated by miR-143/145 cluster in PCa, upregulated genes which were targeted by $m i R-143 / 145$ cluster were analyzed in terms of Kyoto Encyclopedia of Genes and Genomes (KEGG) pathway categories using the GENECODIS program (http://genecodis.cnb.csic.es/). 
Table 1 Patient characteristics

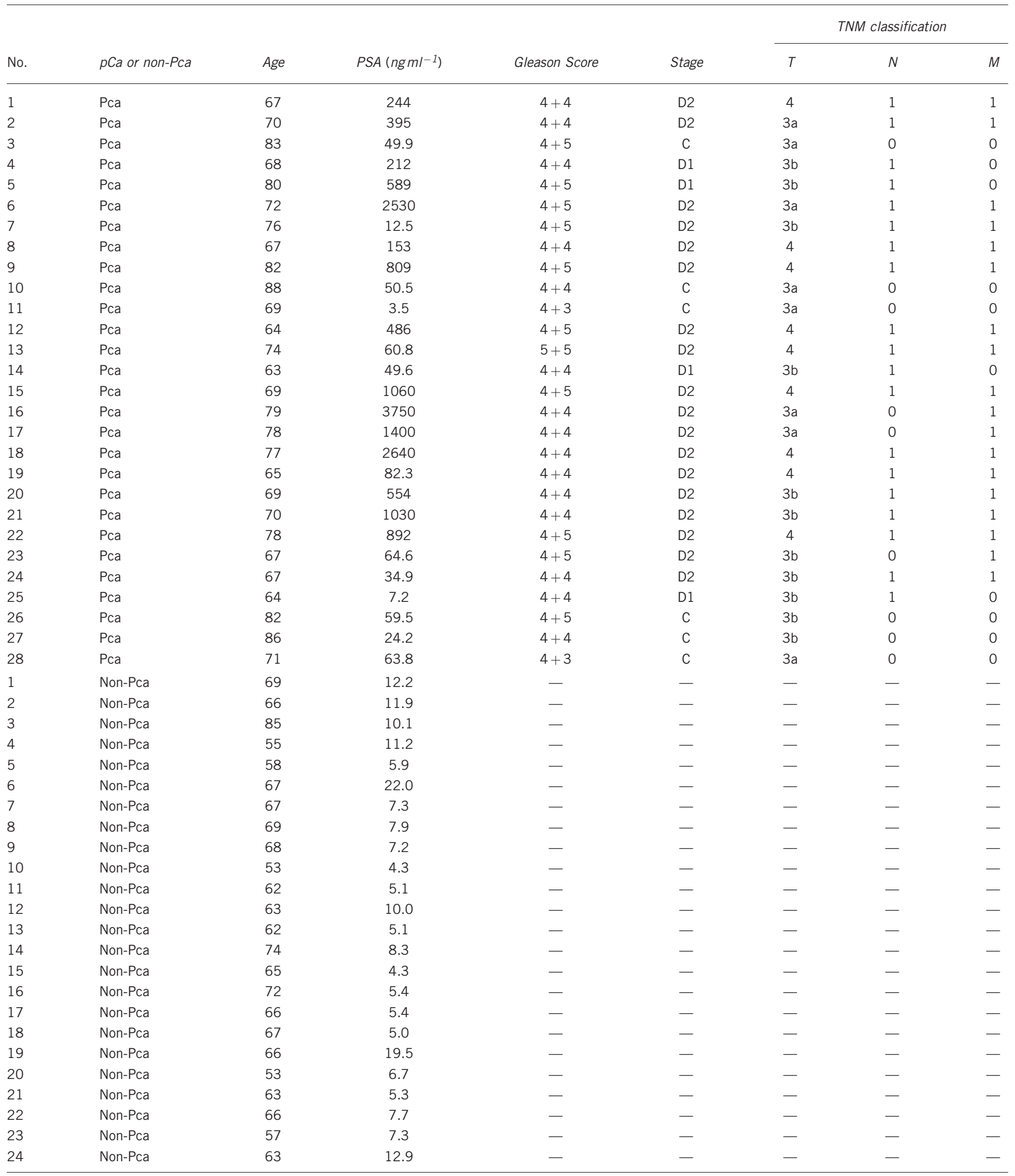

Abbreviations: PCa, prostate cancer; PSA, prostate-specific antigen; TNM, Tumor, Node, Metastatis.

Plasmid construction and dual-luciferase reporter assay Partial wild-type sequences of the GOLM1 $3^{\prime}$ untranslated region (UTR) or those with a deleted miR-143 target site (position 1610-1617 of the GOLM1 $3^{\prime} \mathrm{UTR}$ ) and deleted miR-145 target sites (positions 5-12 and 260-267 of the GOLM1 $3^{\prime}$ UTR) were inserted between the XhoI-PmeI restriction sites in the $3^{\prime} \mathrm{UTR}$ of the hRluc gene in the psiCHECK-2 vector (C8021; Promega, 
Madison, WI, USA). The protocol for vector construction was described previously. ${ }^{18,19}$ Sequences of the oligonucleotides are described in Supplementary Table S1. The synthesized DNA was cloned into the psiCHECK-2 vector. PC3 cells were transfected with $50 \mathrm{ng}$ of the vector and $10 \mathrm{~nm} m i R-143$ or miR-145 using Lipofectamine 2000 (Invitrogen). The activities of firefly and renilla luciferases in cell lysates were determined with a dual-luciferase assay system (E1910; Promega). Normalized data were calculated as the ratio of renilla/firefly luciferase activities.

\section{Immunohistochemistry}

A tissue microarray of 39 prostate tumors and 8 normal prostate tissues was obtained from US Biomax, Inc. (PR956; Rockville, MD, USA). Detailed information on all tumor specimens can be found at http://www.biomax.us/ index.php. The patients' backgrounds and clinicopathological characteristics are summarized in Supplementary Table S2. The tissue microarray was immunostained with an UltraVision Detection System (Thermo Scientific) following the manufacturer's protocol. Primary rabbit polyclonal antibodies against GOLM1 (Sigma-Aldrich) were diluted 1:500. The slides were treated with biotinylated goat antibodies. Diaminobenzidine hydrogen peroxidase was the chromogen, and counterstaining was performed with $0.5 \%$ hematoxylin. Immunostaining was evaluated according to a scoring method described previously. ${ }^{18,21}$ Each case was scored on the basis of the intensity and area of staining. The intensity of staining was graded on the following scale: 0 , no staining; $1+$, mild staining; $2+$, moderate staining; and $3+$, intense staining. The area of staining was evaluated as follows: 0 , no staining of cells in any microscopic fields; $1+,<30 \%$ of cells stained positive; $2+, 30-60 \%$ of cells stained positive; $3+,>60 \%$ of cells stained positive. A combined staining score (intensity + extension) of $<2$ indicated low expression, a score between 3 and 4 indicated moderate expression, and a score between 5 and 6 indicated high expression.

\section{Statistical analysis}

The relationships between two groups and the numerical values obtained by real-time RT-PCR were analyzed using the paired $t$-test. The relationship among three variables and numerical values was analyzed using the Bonferroni-adjusted Mann-Whitney $U$ test. All analyses were performed using Expert StatView software (version 4, SAS Institute Inc., Cary, NC, USA).

\section{RESULTS}

The expression levels of $m i R-143$ and $m i R-145$ in PCa specimens We evaluated the expression levels of $m i R-143$ and $m i R-145$ in normal prostate tissues $(n=24)$ and PCa tissues $(n=28)$. Prostate-specific antigen levels in patients with normal prostate tissues were measured to have a median value of $8.7 \mathrm{ng} \mathrm{ml}^{-1}$ (range $4.3-22 \mathrm{ng} \mathrm{ml}^{-1}$ ), whereas PCa patients had high prostate-specific antigen values, with a median of $182 \mathrm{ng} \mathrm{ml}^{-1}\left(3.4-3750 \mathrm{ng} \mathrm{ml}^{-1}\right)$. PCa patients had progressive disease with $\mathrm{N} 1(67.8 \%)$ and M1 (64.2\%) according to TNM (Tumor, Node, Metastatis) classification (Table 1).

The expression levels of $m i R-143$ and $m i R-145$ were determined by RT-PCR. The expression levels of miR-143 (Figure 1a) and miR-145 (Figure 1b) were significantly lower in PCa tissues compared with normal tissues ( $P=0.00148$ and $P<0.0001$, respectively). The expression of miR-143 was highly correlated with that of miR-145 (correlation coefficient, 0.898; $P<0.0001$; Figure 1c).

Effects of $m i R-143$ and $m i R-145$ transfection on cell proliferation, migration and invasion in PCa cells

Next, the expression levels of miR-143 and miR-145 in DU145 and PC3 cells were determined by RT-PCR and were compared with those of normal prostate tissues. $m i R-143$ and $m i R-145$ were significantly downregulated in DU145 and PC3 cells as compared with normal prostates (Figures 1a and $\mathrm{b}$ ).

To examine the functional roles of $m i R-143$ and $m i R-145$, we performed gain-of-function studies using miRNA transfection into DU145 and PC3 cells. XTT assays revealed significant inhibition of cell proliferation in DU145 and PC3 cells transfected with miR-143 and miR-145 in comparison with mock-transfected cells and control transfectants (cell proliferation, percentage of mock: DU145 cells $68.8 \% \pm 3.7 \%, \quad 53.4 \% \pm 1.1 \%, \quad 100.0 \% \pm 1.3 \%$ and $93.7 \% \pm 1.7 \%$, respectively, $P<0.0001 ;$ PC3 cells $90.4 \% \pm 5.7 \%, 60.0 \% \pm 1.2 \%$, $100.0 \% \pm 3.3 \%$ and $103.2 \% \pm 4.0 \%$, respectively, $P=0.0969$ and $P<0.0005$; Figure 2a).

Moreover, wound-healing assays demonstrated significant inhibition of cell migration in DU145 and PC3 cells transfected with miR143 and miR-145 in comparison with mock-transfected cells and control transfectants (cell migration, percentage of mock: DU145 cells $64.2 \% \pm 5.2 \%, \quad 59.0 \% \pm 10.0 \%, \quad 100.0 \% \pm 7.1 \%$ and $88.7 \% \pm 8.8 \%$, respectively, $P=0.0047$ and $P=0.0016$; PC3 cells $68.9 \% \pm 4.5 \%$, $10.7 \% \pm 4.9 \%, \quad 100.0 \% \pm 5.8 \%$ and $103.6 \% \pm 7.7 \%$, respectively, $P=0.0013$ and $P<0.0001$; Figure $2 \mathrm{~b}$ ).

Similarly, Matrigel invasion assays revealed that transfection with these miRNAs reduced cell invasion. Indeed, the number of invading cells was significantly decreased in DU145 and PC3 cells transfected with miR-143 and miR-145 in comparison with mock-transfected cells and control transfectants (percentage of invading cells: DU145 cells $59.7 \% \pm 7.3 \%, \quad 12.1 \% \pm 2.8 \%, \quad 100.0 \% \pm 4.9 \%$ and $114.1 \% \pm 3.3 \%$, respectively, $P<0.0001 ;$ PC3 cells $90.8 \% \pm 3.2 \%, 17.7 \% \pm 1.9 \%$, $100.0 \% \pm 6.7 \%$ and $103.6 \% \pm 4.2 \%$, respectively, $P=0.1539$ and $P<0.0001$; Figure 2c).
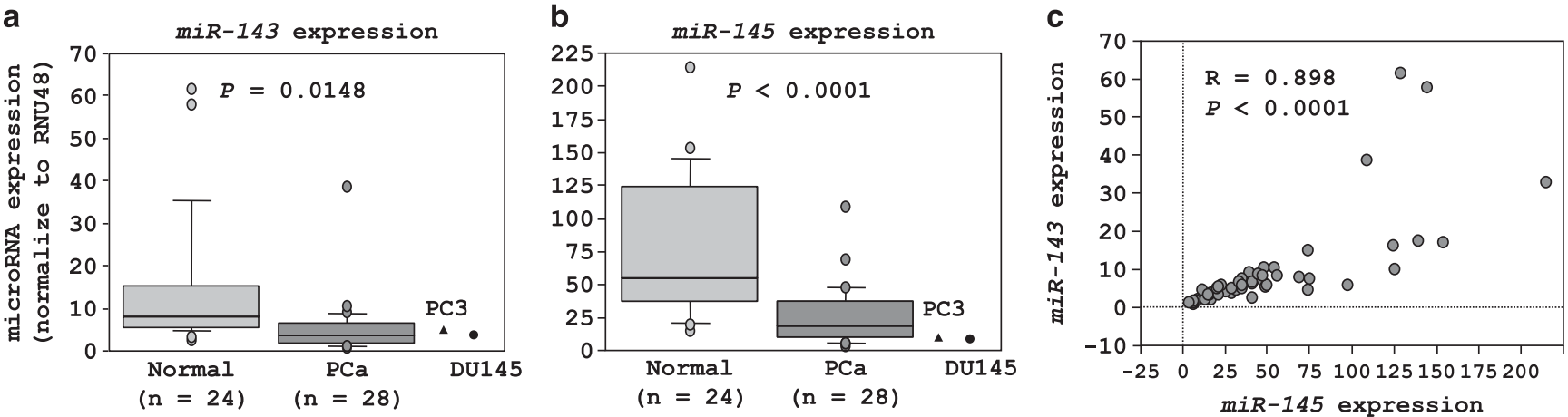

Figure 1 Expression levels of miR-143 and miR-145. (a, b) miR-143 and miR-145 expression levels in clinical PCa specimens and PCa cell lines (PC3 and DU145) compared with non-cancerous tissues. RNU48 was used as the internal control. (c) Correlation between the relative expression level of miR143 ( $x$-axis) and that of miR-145 ( $y$-axis). Levels are plotted as a scatterplot. 

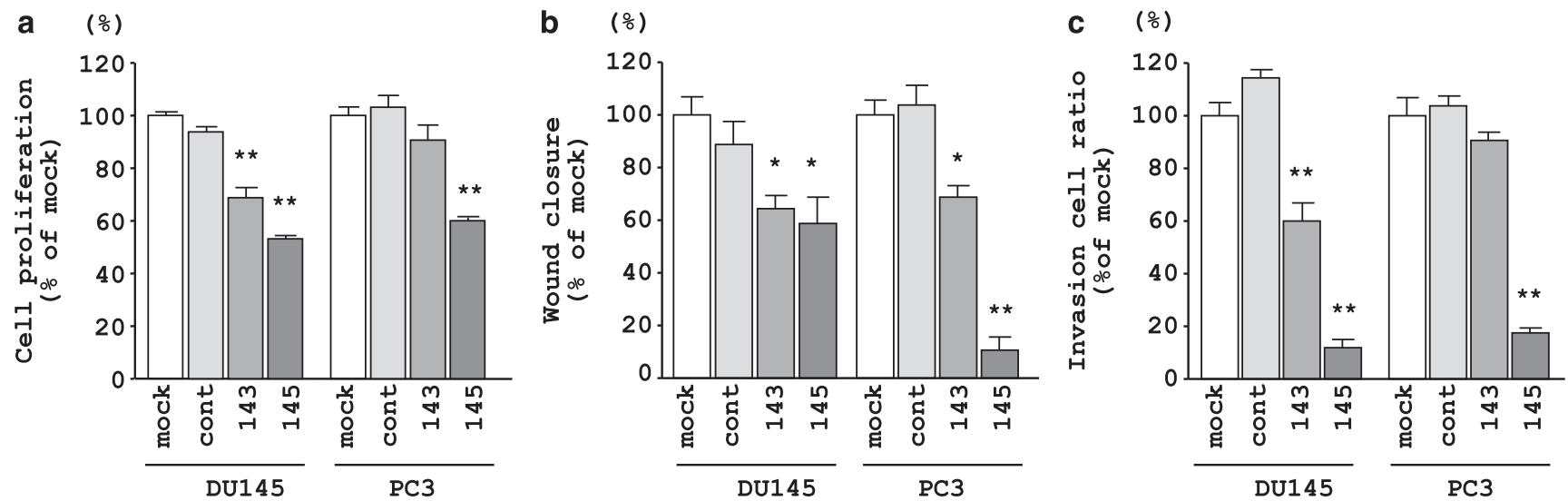

Figure 2 Effects of transfection with mature miR-143 and miR-145 on RCC cell lines. (a) Cell proliferation was determined with XTT assays in PC3 and DU145 cell lines after $72 \mathrm{~h}$ of transfection with $10 \mathrm{~nm}$ miR-143, miR-145, miR-control or mock transfection. (b) Cell migration activity was determined by the wound-healing assay. (c) Cell invasion activity was determined by Matrigel invasion assay. ${ }^{*} P<0.01 ;{ }^{* *} P<0.0001$.

\section{Identification of targets genes regulated by the $m i R-143 / 145$ cluster in PCa}

To gain further insight into the molecular mechanisms and pathways regulated by the tumor-suppressive miR-143/145 cluster in PCa, we performed a combination of gene expression and in silico analyses. The strategy of selection of $m i R-143 / 145$ cluster-regulated genes is shown in Figure 3. The TargetScan program showed that 1053 genes had putative target sites for both miR-143 and miR-145 in their $3^{\prime} \mathrm{UTR}$ regions. To confirm the expression levels of these genes in clinical prostate tissues, GEO database (GEO accession number: GSE29079) analysis was performed. Among the 1053 genes, 347 genes were upregulated in $47 \mathrm{PCa}$ specimens compared with 48 noncancerous prostate tissues. Expression data for the 44 genes $(>1.5$ fold change) are summarized in Table 2, and the other upregulated genes are shown in Supplementary Table S2.

Furthermore, those 347 genes were classified KEGG pathways using GeneCodis program to identify molecular pathways regulated by $m i R$ 143/145 cluster in PCa. Four signaling pathways (endocytosis, oocyte meiosis, protein processing in endoplasmic reticulum and ubiquitinmediated proteolysis) were identified in this analysis (Supplementary Tables S3A-E).

GOLM1 as a target of post-transcriptional repression by the $m i R$ 143/145 cluster in PCa cells

The mRNA and protein expression levels of GOLM1 were markedly downregulated in miR-143- and miR-145-transfected PC3 and DU145 cells in comparison with mock-transfected cells and control transfectants (Figures $4 \mathrm{a}$ and b). Therefore, we next performed a luciferase reporter assay to determine whether GOLM1 mRNA had functional target sites for $m i R-143$ and $m i R-145$. We used three vectors encoding the partial sequences of the $3^{\prime} \mathrm{UTR}$ of GOLM1 mRNA (Figure 4c, Supplementary Table S1). The luminescence intensity was significantly decreased in the presence of two sites targeted by $m i R-143$ or $m i R-145$ (positions $1610-1617$ and 5-12, respectively; Figure $4 \mathrm{c}$, middle and lower panels). In contrast, the luminescence intensity was not decreased by transfection with miR-145 at position 260-267 or with deleted sequences of either miRNA (Figure 4c, middle panel). These data suggested that miR-143 and $m i R-145$ bound directly to specific sites in the $3^{\prime} \mathrm{UTR}$ of GOLM1 mRNA.
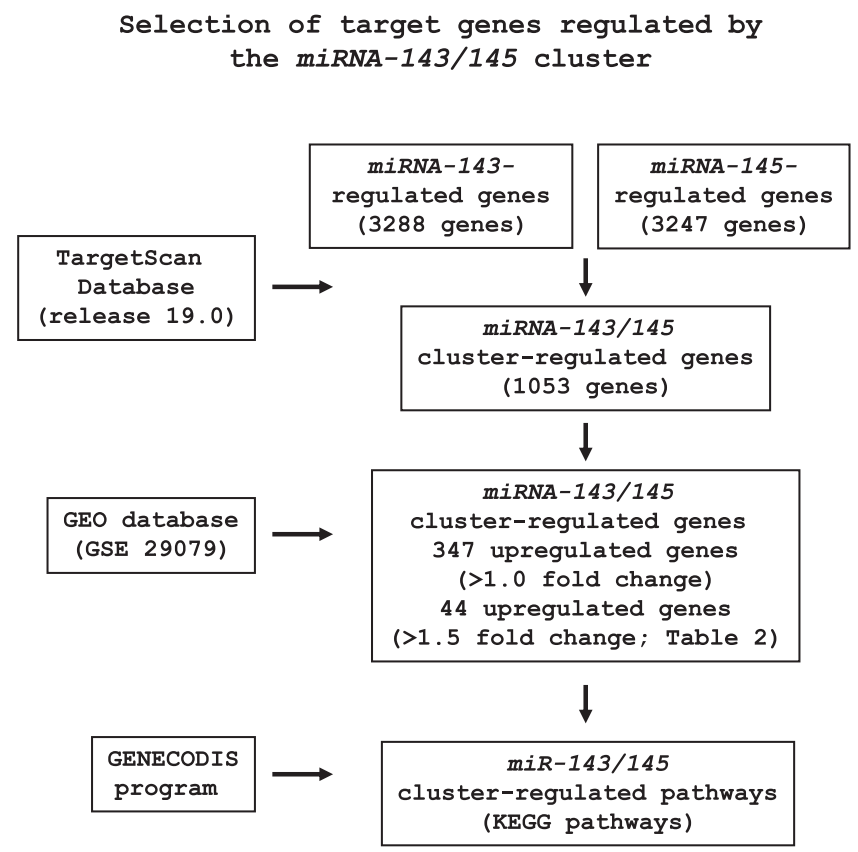

Figure 3 Flow chart of the strategy for analysis of miR-143/145 cluster target genes. A total of 1053 genes were selected as putative miR-143/145 cluster target genes by TargetScan database analysis. We then analyzed the expression levels of these 1053 genes by using available data sets of GEO (GSE 29079). The analyses showed that 44 genes were significantly upregulated in PCa specimens compared with normal specimens.

Effects of GOLM1 knockdown on cell proliferation, migration and invasion in PCa cell lines

The expression of GOLM1 mRNA was upregulated in DU145 and PC3 cells compared with normal prostate tissues (data not shown). Therefore, to examine the functional role of GOLM1 in PCa, we performed loss-of-function studies using transfection with two different siRNA sequences targeting GOLM1 in DU145 and PC3 cells. The expression of GOLM1 mRNA and protein was markedly repressed in si-GOLM1 transfectants (Figures 5a and b).

XTT assays revealed that transfection with si-GOLM1 inhibited cell proliferation in PC3 cells compared with mock-transfected cells and si-control transfectants; however, no significant inhibition was 
Table 2 Identification of target genes regulated by the miR-143/145 cluster in PCa

\begin{tabular}{|c|c|c|c|}
\hline Entrez gene ID & Gene symbol & Fold change & Description \\
\hline 51280 & GOLM1 & 3.52 & Golgi membrane protein 1 \\
\hline 28996 & HIPK2 & 2.35 & Homeodomain-interacting protein kinase 2 \\
\hline 54848 & ARHGEF38 & 2.26 & Rho guanine nucleotide exchange factor (GEF) 38 \\
\hline 4646 & MY06 & 2.17 & Myosin VI \\
\hline 56894 & AGPAT3 & 2.02 & 1-Acylglycerol-3-phosphate 0-acyltransferase 3 \\
\hline 3069 & HDLBP & 1.95 & High-density lipoprotein-binding protein \\
\hline 51114 & ZDHHC9 & 1.92 & Zinc finger, DHHC-type containing 9 \\
\hline 7764 & ZNF217 & 1.90 & Zinc finger protein 217 \\
\hline 7941 & PLA2G7 & 1.90 & Phospholipase A2, group VII \\
\hline 19 & $\mathrm{ABCA} 1$ & 1.80 & ATP-binding cassette, sub-family A ( $\mathrm{ABC} 1$ ), member 1 \\
\hline 11057 & ABHD2 & 1.80 & Abhydrolase domain containing 2 \\
\hline 27075 & TSPAN13 & 1.79 & Tetraspanin 13 \\
\hline 22848 & AAK1 & 1.79 & AP2-associated kinase 1 \\
\hline 51313 & FAM198B & 1.78 & Family with sequence similarity 198 , member B \\
\hline 23200 & ATP11B & 1.78 & ATPase, class VI, type 11B \\
\hline 2065 & ERBB3 & 1.72 & V-erb-b2 avian erythroblastic leukemia viral oncogene homolog 3 \\
\hline 206358 & SLC36A1 & 1.72 & Solute carrier family 36 (proton/amino acid symporter), member 1 \\
\hline 84914 & ZNF587 & 1.71 & Zinc finger protein 587 \\
\hline 79939 & SLC35E1 & 1.70 & Solute carrier family 35 , member E1 \\
\hline 3480 & IGF1R & 1.69 & Insulin-like growth factor 1 receptor \\
\hline 2335 & FN1 & 1.68 & Fibronectin 1 \\
\hline 117177 & RAB3IP & 1.66 & RAB3A-interacting protein \\
\hline 23362 & PSD3 & 1.65 & Pleckstrin and Sec7 domain containing 3 \\
\hline 57182 & ANKRD50 & 1.64 & Ankyrin repeat domain 50 \\
\hline 1729 & DIAPH1 & 1.64 & Diaphanous-related formin 1 \\
\hline 9517 & SPTLC2 & 1.63 & Serine palmitoyltransferase, long chain base subunit 2 \\
\hline 26999 & CYFIP2 & 1.63 & Cytoplasmic FMR1-interacting protein 2 \\
\hline 3340 & NDST1 & 1.62 & N-deacetylase/N-sulfotransferase (heparan glucosaminyl) 1 \\
\hline 9931 & HELZ & 1.60 & Helicase with zinc finger \\
\hline 22834 & ZNF652 & 1.58 & Zinc finger protein 652 \\
\hline 3488 & IGFBP5 & 1.56 & Insulin-like growth factor binding protein 5 \\
\hline 27102 & EIF2AK1 & 1.55 & Eukaryotic translation initiation factor 2-alpha kinase 1 \\
\hline 8243 & SMC1A & 1.55 & Structural maintenance of chromosomes $1 \mathrm{~A}$ \\
\hline 7311 & UBA52 & 1.55 & Ubiquitin A-52 residue ribosomal protein fusion product 1 \\
\hline 1601 & DAB2 & 1.53 & Dab, mitogen-responsive phosphoprotein, homolog 2 (Drosophila) \\
\hline 11060 & WWP2 & 1.53 & WW domain containing E3 ubiquitin protein ligase 2 \\
\hline 26472 & PPP1R14B & 1.52 & Protein phosphatase 1 , regulatory (inhibitor) subunit 14B \\
\hline 54765 & TRIM44 & 1.52 & Tripartite motif containing 44 \\
\hline 57493 & HEG1 & 1.52 & Heart development protein with EGF-like domains 1 \\
\hline 2035 & EPB41 & 1.51 & Erythrocyte membrane protein band 4.1 (elliptocytosis $1, \mathrm{RH}$-linked) \\
\hline 79822 & ARHGAP28 & 1.51 & Rho GTPase activating protein 28 \\
\hline 2806 & GOT2 & 1.51 & Glutamic-oxaloacetic transaminase 2, mitochondrial \\
\hline 6801 & STRN & 1.51 & Striatin, calmodulin-binding protein \\
\hline 57619 & SHROOM3 & 1.51 & Shroom family member 3 \\
\hline
\end{tabular}

Abbreviation: PCa, prostate cancer.

observed in DU145 cells (cell proliferation, percentage of mock: DU145 cells $89.5 \% \pm 2.3 \%, \quad 92.7 \% \pm 2.9 \%, \quad 100.0 \% \pm 1.9 \%$ and $98.5 \% \pm 1.7 \%$, respectively, $P=0.0033$ and $P=0.031$; PC3 cells $78.0 \% \pm 1.2 \%, \quad 81.5 \% \pm 0.9 \%, \quad 100.0 \% \pm 1.3 \%$ and $92.0 \% \pm 1.4 \%$, respectively, $P<0.0001$, Figure 6a).

Wound-healing assays demonstrated that transfection with the two siRNAs targeting GOLM1 significantly inhibited cell migration compared with mock-transfected cells and control transfectants in PC3 and DU145 cells (cell migration, percentage of mock: DU145 cells $\quad 68.5 \% \pm 7.3 \%, \quad 43.4 \% \pm 4.6 \%, \quad 100.0 \% \pm 17.7 \% \quad$ and $115.6 \% \pm 4.5 \%$, respectively, $P=0.0389$ and $P=0.0008$; PC3 cells $39.9 \% \pm 4.7 \%, \quad 27.8 \% \pm 4.7 \%, \quad 100.0 \% \pm 2.1 \%$ and $105.1 \% \pm 8.2 \%$, respectively, $P<0.0001$; Figure $6 \mathrm{~b}$ ).
Similarly, Matrigel invasion assays demonstrated that the number of invading cells was significantly decreased si-GOLM1-1 and si-GOLM1-2 transfectants compared with mock-transfected cells and control transfectants, especially in PC3 cells (percentage of invading cells: DU145 cells $70.5 \% \pm 5.4 \%, \quad 80.9 \% \pm 5.5 \%$, $100.0 \% \pm 4.4 \%$ and $127.1 \% \pm 7.0 \%$, respectively, $P=0.0022$ and $P=0.0399 ;$ PC3 cells $39.7 \% \pm 2.6 \%, 8.8 \% \pm 1.0 \%, 100.0 \% \pm 14.8 \%$ and $67.6 \% \pm 9.2 \%$, respectively, $P<0.0001$; Figure $6 c$ ).

\section{Immunohistochemistry for detection of GOLM1 in a PCa tissue microarray}

Next, we determined the expression levels of GOLM1 in PCa tissues and normal prostate tissues by immunohistochemical staining. 

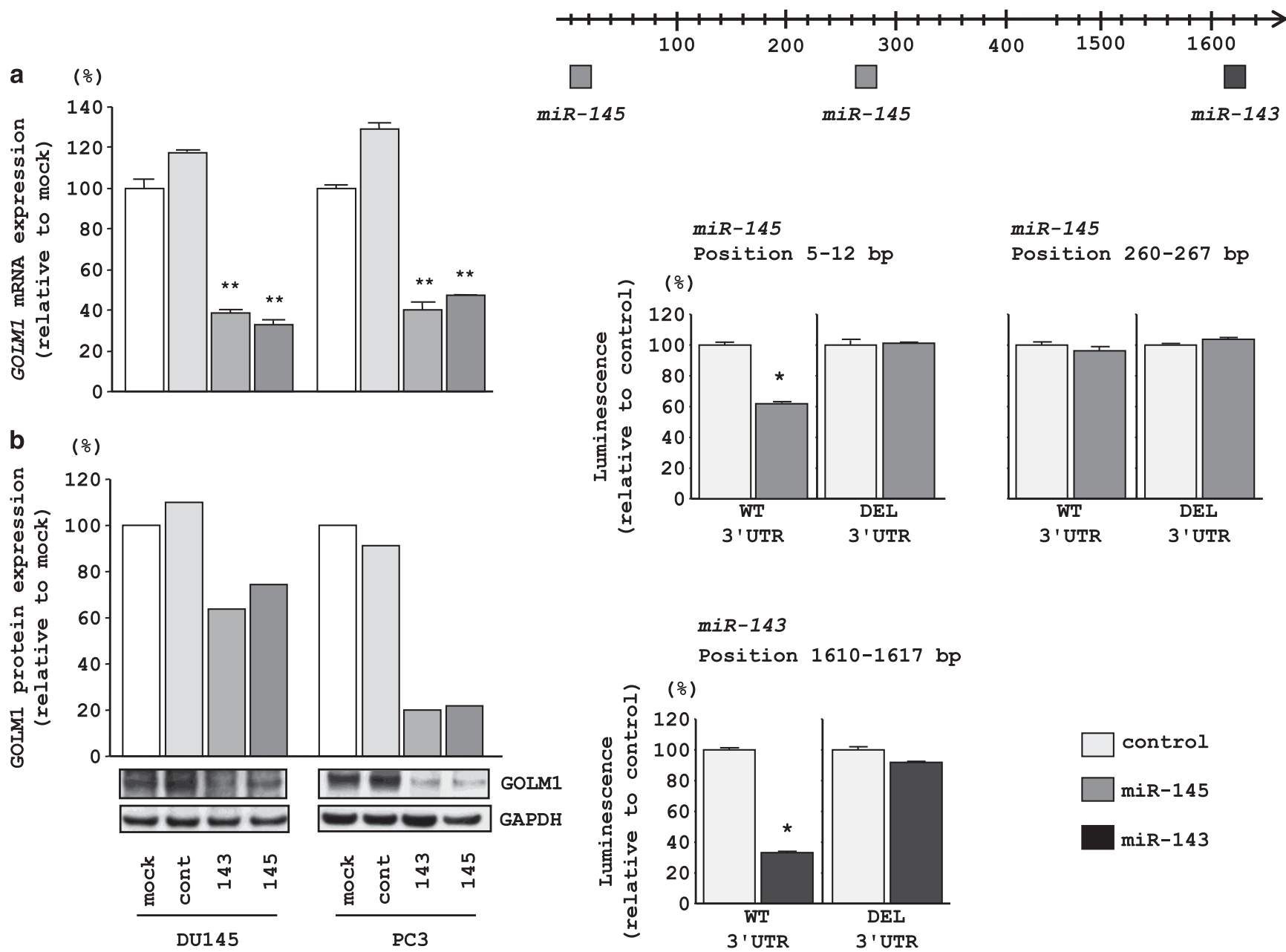

Figure 4 GOLM1 expression was suppressed by miR-143 and miR-145 transfection at both the mRNA and protein levels in DU145 and PC3 cells. (a) GOLM1 mRNA expression $72 \mathrm{~h}$ after transfection with miR-143 and miR-145. GAPDH expression was used for normalization. ${ }^{* *} P<0.0001$. (b) GOLM1 protein expression $72 \mathrm{~h}$ after transfection with miR-143 and miR-145. GAPDH was used as a loading control. (c) miR-143 and miR-145 binding sites in the 3'UTR of GOLM1 mRNA. Luciferase reporter assay using the three vectors encoding putative miR-145 target sites at positions 5-12 and 260-267 and the putative miR-143 target site at position 1610-1617 for both wild type (WT) and deletion (DEL). Renilla luciferase values were normalized to firefly luciferase values. ${ }^{*} P<0.01$

GOLM1 was strongly expressed in several tumor lesions (Figures 7a and b), whereas no or low expression was observed in normal tissues (Figure 7c). Moreover, the expression score for GOLM1 protein was significantly higher in PCa tissues than in normal tissues $(P=0.0244$, Figure $7 \mathrm{~d})$. The patients' backgrounds and clinicopathological characteristics are summarized in Supplementary Table S4. There were no significant correlations between in GOLM1 expression and the tested clinicopathological parameters (Gleason Score and stages, data not shown).

\section{DISCUSSION}

Recent studies of our miRNA expression signatures, including PCa, demonstrated that miR-143/145 cluster was frequently reduced in cancer tissues. ${ }^{9,10,18,19}$ Therefore, it is suggested that the miR-143/145 cluster have an important tumor-suppressive function in human cancers. In this study, we first tested the expression levels of miR-143 and $m i R-145$ in needle biopsy core tissues collected from patients with $\mathrm{PCa}$ and non-PCa. We confirmed the reduced expression of both
miRNAs in $\mathrm{PCa}$, and expression analysis showed positive correlations between $m i R-143$ and $m i R-145$. These data suggested that $m i R-143$ and $m i R-145$ are regulated by the same regulatory systems, and silencing of this miRNA cluster may be a key step in the oncogenesis and progression of PCa. Moreover, miR-143 and miR-145 were reported to inhibit stem cell characteristics of PC3 cells, resulting in the inhibition of bone invasion and tumorigenicity of PC3 cells in vivo. ${ }^{12}$ Others have reported that wild-type p53 suppresses the epithelial-mesenchymal transition and stemness of PC3 cells by modulating miR-145. ${ }^{13}$ Tumor-suppressor $\mathrm{p} 53$ has been reported to transcriptionally regulate $m i R-145$, inducing its expression by interaction with a potential p53 response element at the miR-145 promoter. ${ }^{22}$ Interestingly, c-MYC is directly repressed by miR-145. These studies indicate that tumor-suppressive $m i R-145$, as a new member of the p53 regulatory network, contributes to the direct linkage between p53 and c-MYC in human cancer pathways. ${ }^{23}$ Recent studies have shown that the miR-143/145 cluster is repressed by RAS responsive element-binding protein (RREB1) downstream of 

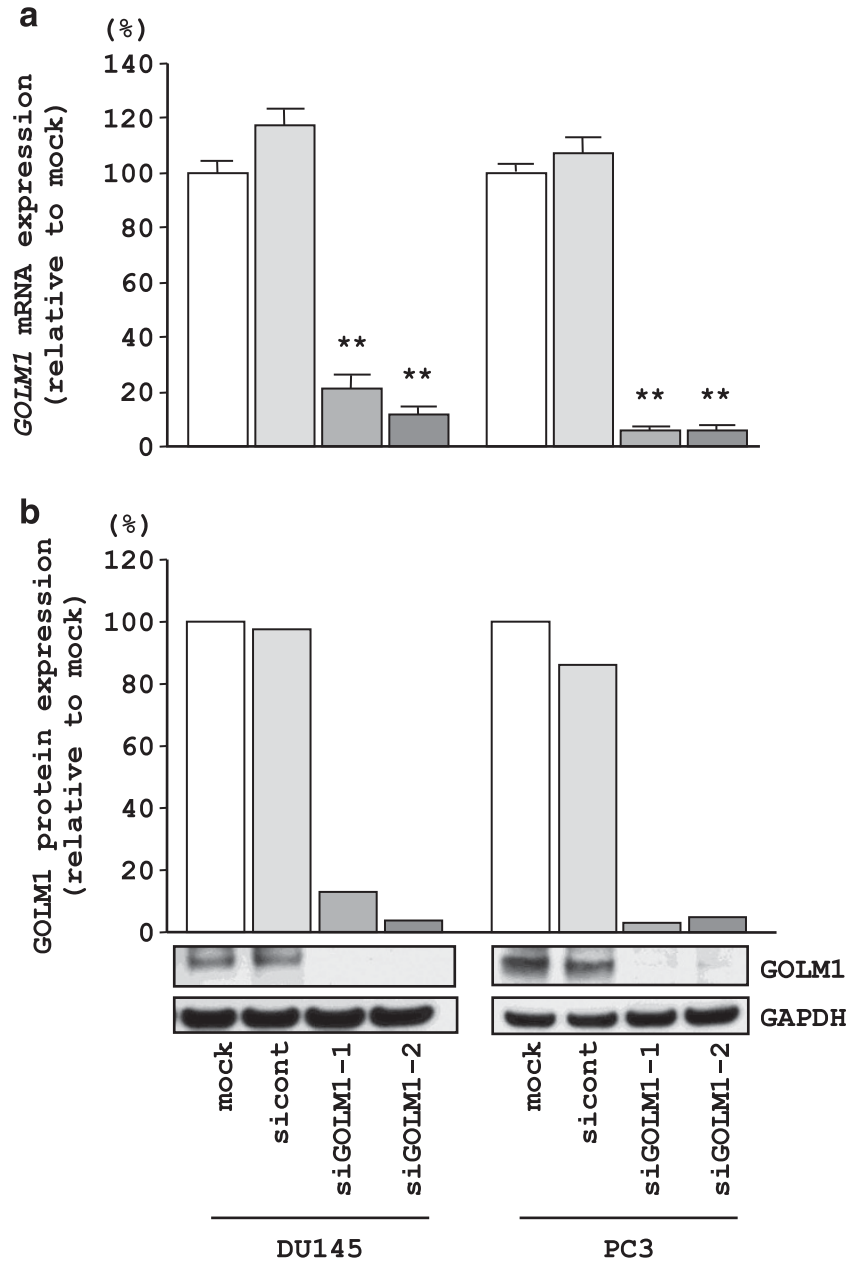

Figure 5 GOLM1 mRNA and protein expression levels were suppressed by si-GOLM1 transfection in DU145 and PC3 cells. (a) GOLM1 mRNA expression $72 \mathrm{~h}$ after transfection with si-GOLM1. GAPDH expression was used for normalization. ${ }^{* *} P<0.0001$. (b) GOLM1 protein expression $72 \mathrm{~h}$ after transfection with si-GOLM1. GAPDH was used as a loading control. The ratio of GOLM1/GAPDH expression was evaluated using ImageJ software (ver. 1.43; http://rsbweb.nih.gov/ij/index.html).

constitutively active KRAS. ${ }^{24}$ Thus, investigation of molecular targets and cancer pathways regulated by the $m i R-143 / 145$ cluster is necessary for the clinical development of novel cancer therapeutics.

Researchers are interested in investigating the functional significance of the miR-143/145 cluster in cancer cells and identifying novel targets commonly regulated by these clustered miRNAs in human cancers, including PCa. Multiple reports have shown that miR-143 and miR-145 function as tumor suppressors, targeting a variety of oncogenic genes in several types of cancers. ${ }^{25,26}$ Our recent data demonstrated that $m i R-143 / 145$ cluster inhibited cancer cell migration and invasion in renal cell carcinoma cells via targeting hexokinase-2, suggesting that this cluster acts as a tumor suppressor in renal cell carcinoma. ${ }^{27}$ In this study, we also showed that $m i R-143 / 145$ cluster function as a tumor suppressor in PCa. Therefore, identification of miR-143/145 cluster-regulated cancer pathways may provide new insights into the molecular mechanisms of human cancers.

We hypothesize that identification of novel cancer pathways and target genes regulated by tumor-suppressive miRNAs is an important first step in understanding human oncogenesis. Based on this view, we performed a combination study of genome-wide gene expression analysis in cells transfected with tumor-suppressive miRNAs, in silico analysis and identification of novel miRNA-regulated cancer pathways and targets, as reported previously. ${ }^{17,18}$ In the present study, we identified the GOLM1 as a miR-143/145 cluster-regulated molecular target and proved that miR-143/145 cluster directly binding $3^{\prime} \mathrm{UTR}$ of GOLM1. We also found that silencing of GOLM1 resulted in significant inhibition of migration and invasion in PCa cells. Furthermore, overexpression of GOLM1 was observed in PCa clinical specimens, as detected by immunohistochemistry. These data suggested that GOLM1 functioned as an oncogene, contributing to cancer cell migration and invasion. However, the mechanisms mediating the overexpression of GOLM1 in PCa and its role in cancer cell migration and invasion remain unclear.

The GOLM1/GP73/GOLPH2 protein is encoded by the GOLM1 gene, located on human chromosome 9q21.33. GOLM1 contains two transcript variants, and both variants encode the same protein. ${ }^{14}$ The expression of GOLM1 has been shown to be overexpressed in human $\mathrm{PCa}$ tissue,lung adenocarcinoma and hepatocellular carcinoma. ${ }^{15-17,28,29}$ Structurally, GOLM1 resembles a type II Golgi transmembrane protein, and studies have shown that GOLM1 processes proteins synthesized in the rough endoplasmic reticulum and assists in the transport of protein cargo through the Golgi apparatus. $^{30}$ In PCa cells, GOLM1 is localized in the subapical cytoplasmic region, typical of Golgi distribution. Interestingly, the expression of GOLM1 is higher in the blood of patients with hepatocellular carcinoma and in the urine of patients with $\mathrm{PCa}$ than in healthy individuals, suggesting that GOLM1 is a useful tool for the diagnosis of human cancers. ${ }^{14-17,29}$

However, despite that a number of studies have demonstrated GOLM1 expression in cancers, we questioned whether GOLM1 contributed to the oncogenesis and metastasis of PCa. According to our immunohistochemistry data, there was no correlation between GOLM1 protein expression and tumor grade or stage. Even the gene expression data (GSE 29079) was not able to find clinical correlation. In contrast, another study reported the moderately increased expression of GOLM1 in prostatic intra-epithelial neoplasia, a precancerous condition. ${ }^{15}$ This data suggested that GOLM1 contributes to the oncogenesis and progression of PCa. The clinical study with expression status of GOLM1 and clinical features of PCa will be necessary in future.

Multiple reports had indicated that the Golgi apparatus is a key player in cell migration and acts as a hub for different signaling pathways, including the mitogen-activated protein kinase pathway, the mammalian target of rapamycin pathway, Rho family GTPases and cyclin-dependent kinases, through signaling crucial molecules to translocate to and from the Golgi, thereby playing a key role in cell migration and invasion. ${ }^{31,32}$ Therefore, elucidation of the regulatory networks of the Golgi apparatus will provide important information for the development of new therapeutic strategies against cancer cell metastasis. Further research is needed to reveal the oncogenic functions of GOLM1 that are regulated by the tumor-suppressive miR-143/145 cluster in PCa. Therefore, improving our understanding of GOLM1 signaling, as mediated by the tumor-suppressive $m i R-143 / 145$ cluster, should shed light on the mechanisms of PCa oncogenesis and metastasis and assist in the development of more effective therapeutic intervention for this disease.

In conclusion, downregulation of the $m i R-143 / 145$ cluster is frequently observed in PCa clinical specimens. Restoration of these miRNAs significantly inhibited cancer cell migration and invasion, suggesting that $m i R-143 / 145$ functioned as a tumor suppressor in $\mathrm{PCa}$. To the best of our knowledge, this is the first report 

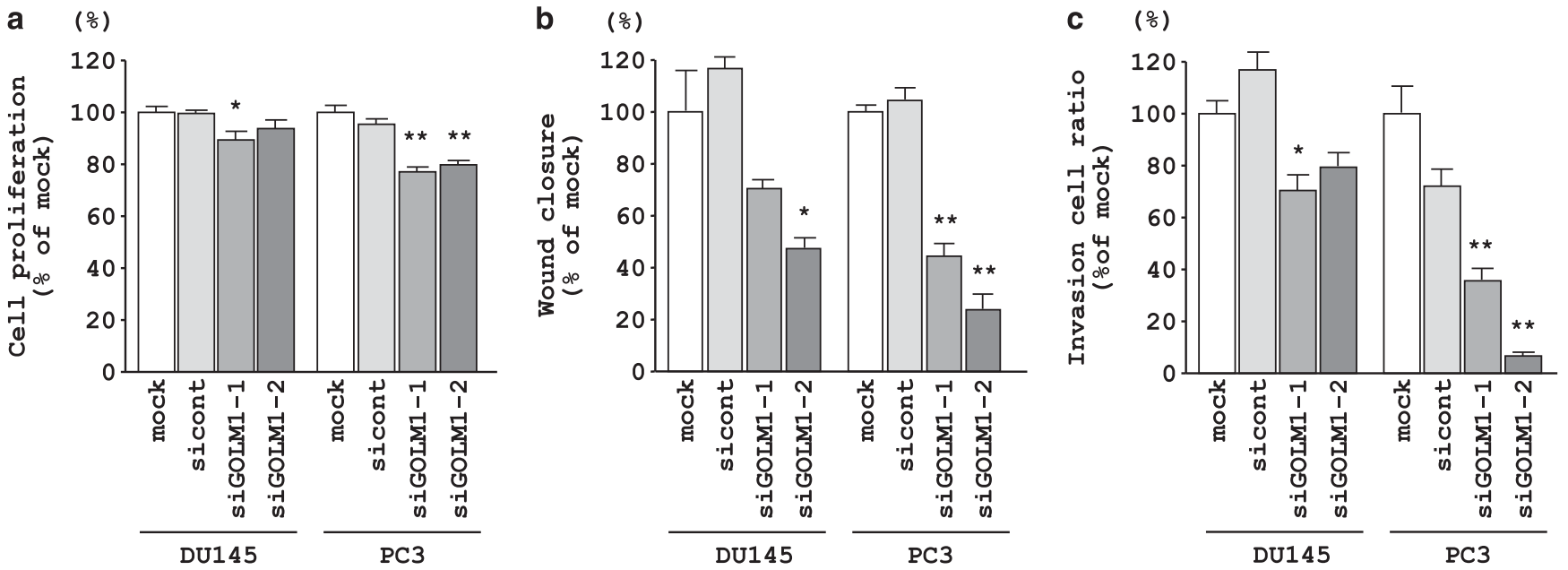

Figure 6 Effects of GOLM1 silencing by si-GOLM1 transfection on the PCa cells. (a) Cell proliferation was determined by the XTT assay. (b) Cell migration activity was determined by the wound-healing assay. (c) Cell invasion activity was determined by the Matrigel invasion assay. ${ }^{* *} P<0.0001,{ }^{*} P<0.01$.
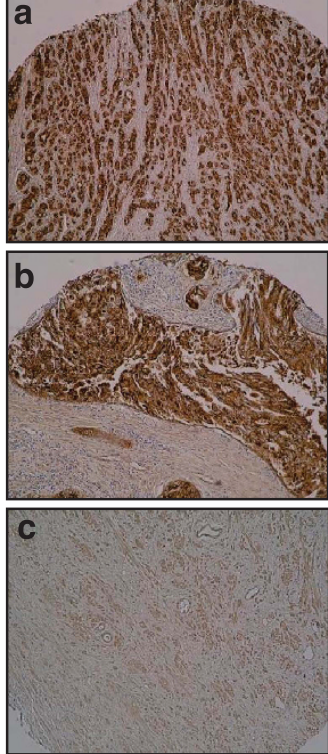

$\mathbf{x} 100$
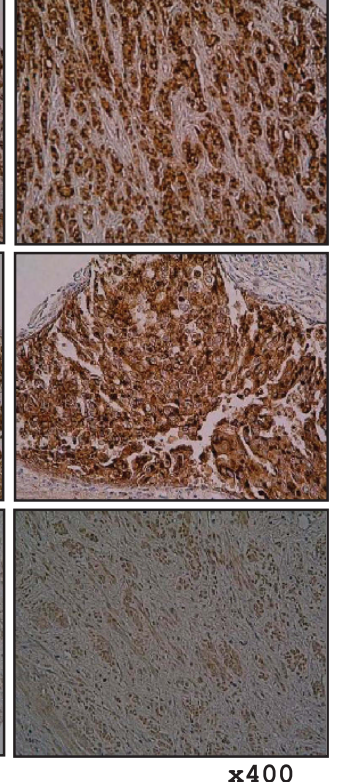

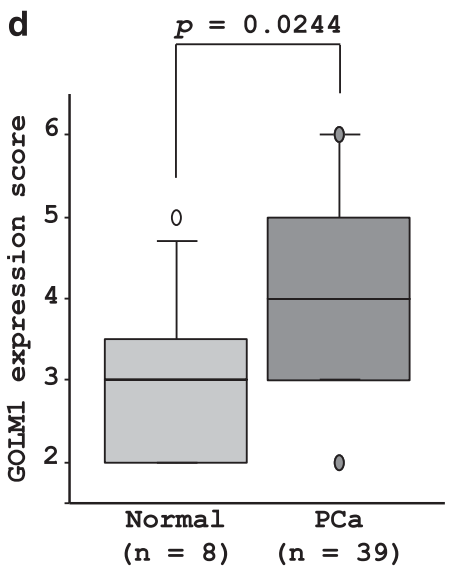

Figure 7 Immunohistochemical staining of GOLM1 in PCa $(n=39)$ and normal prostate tissues $(n=8)$ by tissue microarray (left panel, original magnification: $\times 100$; right panel, original magnification: $\times 400$ ). (a) A strongly stained tumor lesion (Gleason Score $4+5=9$, pT3bN1M0). (b) A strongly stained tumor lesion (Gleason Score $4+5=9$, pT3bNOMO). (c) Negative staining in normal, non-malignant prostate tissues. (d) Quantification of GOLM1 expression. The expression of GOLM1 was upregulated in PCa specimens compared with normal prostate tissues $(P=0.00244)$.

demonstrating that the tumor-suppressive miR-143/145 cluster directly regulated GOLM1 in PCa cells. Moreover, GOLM1 was upregulated in PCa clinical specimens and contributed to cancer cell migration and invasion, indicating that this protein functioned as an oncogene. The identification of novel target oncogenes regulated by the miR-143/145 cluster may lead to a better understanding of PCa oncogenesis and the development of new therapeutic strategies to treat this disease.

\section{CONFLICT OF INTEREST}

All the authors declare no conflict of interest.

\section{ACKNOWLEDGEMENTS}

This study was supported by the KAKENHI (C), 24592590 and (C), 23592351.
1 Siegel, R., Naishadham, D. \& Jemal, A. Cancer statistics, 2013. CA Cancer J. Clin. 63 11-30 (2013).

2 Bott, S. R., Birtle, A. J., Taylor, C. J. \& Kirby, R. S. Prostate cancer management: 2. An update on locally advanced and metastatic disease. Postgrad. Med. J. 79, 643-645 (2003).

3 Timsit, M. O., Lebret, T. \& Mejean, A. Chemotherapy of hormonorefractory and hormonoresistant metastatic prostate cancer. Prog. Urol. 18(Suppl 7), S365-S375 (2008)

4 Carthew, R. W. \& Sontheimer, E. J. Origins and mechanisms of miRNAs and siRNAs. Cell 136, 642-655 (2009).

5 Filipowicz, W., Bhattacharyya, S. N. \& Sonenberg, N. Mechanisms of post-transcriptional regulation by microRNAs: are the answers in sight? Nat. Rev. Genet. 9, 102-114 (2008)

6 Friedman, R. C., Farh, K. K., Burge, C. B. \& Bartel, D. P. Most mammalian mRNAs are conserved targets of microRNAs. Genome Res. 10, 92-105 (2009).

7 Hobert, O. Gene regulation by transcription factors and microRNAs. Science $\mathbf{3 1 9}$, 1785-1786 (2008) 
8 Iorio, M. V. \& Croce, C. M. MicroRNAs in cancer: small molecules with a huge impact. J. Clin. Oncol. 27, 5848-5856 (2009).

9 Fuse, M., Kojima, S., Enokida, H., Chiyomaru, T., Yoshino, H., Nohata, N. et al. Tumor suppressive microRNAs (miR-222 and miR-31) regulate molecular pathways based on microRNA expression signature in prostate cancer. J. Hum. Genet. 57, 691-699 (2012).

10 Kano, M., Seki, N., Kikkawa, N., Fujimura, L., Hoshino, I., Akutsu, Y. et al. miR-145, miR-133a and miR-133b: tumor suppressive miRNAs target FSCN1 in esophageal squamous cell carcinoma. Int. J. Cancer 127, 2804-2814 (2010).

11 Fuse, M., Nohata, N., Kojima, S., Sakamoto, S., Chiyomaru, T., Kawakami, K. et al. Restoration of miR-145 expression suppresses cell proliferation, migration and invasion in prostate cancer by targeting FSCN1. Int. J. Oncol. 38, 1093-1101 (2011).

12 Huang, S., Guo, W., Tang, Y., Ren, D., Zou, X. \& Peng, X. miR-143 and miR-145 inhibit stem cell characteristics of PC-3 prostate cancer cells. Oncol. Rep. 28, 1831-1837 (2012).

13 Guo, W., Ren, D., Chen, X., Tu, X., Huang, S., Wang, M. et al. HEF1 promotes epithelial mesenchymal transition and bone invasion in prostate cancer under the regulation of microRNA-145. J. Cell Biochem. 114, 1606-1615 (2013).

14 Kim, H. J., Lv, D., Zhang, Y., Peng, T. \& Ma, X. Golgi phosphoprotein 2 in physiology and in diseases. Cell Biosci. 2, 31 (2012).

15 Varambally, S., Laxman, B., Mehra, R., Cao, Q., Dhanasekaran, S. M., Tomlins, S. A. et al. Golgi protein GOLM1 is a tissue and urine biomarker of prostate cancer. Neoplasia 10, 1285-1294 (2008).

16 Kristiansen, G., Fritzsche, F. R., Wassermann, K., Jager, C., Tolls, A., Lein, M. et al. GOLPH2 protein expression as a novel tissue biomarker for prostate cancer: implications for tissue-based diagnostics. Br. J. Cancer 99, 939-948 (2008).

$17 \mathrm{Li}$, W., Wang, X., Li, B., Lu, J. \& Chen, G. Diagnostic significance of overexpression of Golgi membrane protein 1 in prostate cancer. Urology 80, 952 e1-7 (2012).

18 Yoshino, H., Chiyomaru, T., Enokida, H., Kawakami, K., Tatarano, S., Nishiyama, K. et al. The tumour-suppressive function of miR-1 and miR-133a targeting TAGLN2 in bladder cancer. Br. J. Cancer 104, 808-818 (2011).

19 Nohata, N., Hanazawa, T., Kikkawa, N., Sakurai, D., Fujimura, L., Chiyomaru, T. et al. Tumour suppressive microRNA-874 regulates novel cancer networks in maxillary sinus squamous cell carcinoma. Br. J. Cancer 105, 833-841 (2011).

20 Brase, J. C., Johannes, M., Mannsperger, H., Falth, M., Metzger, J., Kacprzyk, L. A et al. TMPRSS-ERG-specific transcriptional modulation is associated with prostate cancer biomarkers and TGF- $\beta$ signaling. BMC Cancer 11, 507 (2011).
21 Kojima, S., Chiyomaru, T., Kawakami, K., Yoshino, H., Enokida, H., Nohata, N. et al. Tumour suppressors miR-1 and miR-133a target the oncogenic function of purine nucleoside phosphorylase (PNP) in prostate cancer. Br. J. Cancer 106, 405-413 (2012).

22 Jain, A. K., Allton, K., lacovino, M., Mahen, E., Milczarek, R. J., Zwaka, T. P. et al. p53 regulates cell cycle and microRNAs to promote differentiation of human embryonic stem cells. PLoS Biol. 10, e1001268 (2012).

23 Sachdeva, M., Zhu, S., Wu, F., Wu, H., Walia, V., Kumar, S. et al. p53 represses C-Myc through induction of the tumor suppressor miR-145. Proc. Natl Acad. Sci. USA 106, 3207-3212 (2009).

24 Kent, O. A., Chivukula, R. R., Mullendore, M., Wentzel, E. A., Feldmann, G., Lee, K. H. et al. Repression of the miR-143/145 cluster by oncogenic Ras initiates a tumor-promoting feed-forward pathway. Genes Dev. 24, 2754-2759 (2010).

25 Sachdeva, M. \& Mo, Y. Y. miR-145-mediated suppression of cell growth, invasion and metastasis. Am. J. Transl. Res. 2, 170-180 (2010).

26 Yoshino, $\mathrm{H}$., Seki, N., Itesako, T., Chiyomaru, T., Nakagawa, M. \& Enokida, $\mathrm{H}$. Abberant expression of microRNAs in bladder cancer. Nat. Rev. Urol. 10, 396-404 (2013).

27 Yoshino, H., Enokida, H., Itesako, T., Kojima, S., Kinoshita, T., Tatarano, S. et al. The tumor-suppressive microRNA-143/145 cluster targets hexokinase-2 in renal cell carcinoma. Cancer Sci. doi:10.1111/cas.12282 (2013).

28 Zhang, F., Gu, Y., Li, X., Wang, W., He, J. \& Peng, T. Up-regulated Golgi phosphoprotein 2 (GOLPH2) expression in lung adenocarcinoma tissue. Clin. Biochem. 43, 983-991 (2010).

29 Bachert, C., Fimmel, C. \& Linstedt, A.D. Endosomal trafficking and proprotein convertase cleavage of cis Golgi protein GP73 produces marker for hepatocellular carcinoma. Traffic 8, 1415-1423 (2007).

30 Kladney, R. D., Bulla, G. A., Guo, L., Mason, A. L., Tollefson, A. E., Simon, D. J. et al. GP73, a novel Golgi-localized protein upregulated by viral infection. Gene $\mathbf{2 4 9}, 53-65$ (2000).

31 Migita, T. \& Inoue, S. Implications of the Golgi apparatus in prostate cancer. Int. J. Biochem. Cell Biol. 44, 1872-1876 (2012).

32 Millarte, V. \& Farhan, H. The Golgi in cell migration: regulation by signal transduction and its implications for cancer cell metastasis. ScientificWorldJournal 2012, 498278 (2012).

Supplementary Information accompanies the paper on Journal of Human Genetics website (http://www.nature.com/jhg) 